\title{
Automatic Image-Based Cardiac and Respiratory Cycle Synchronization and Gating of Image Sequences
}

\author{
Hari Sundar, Ali Khamene, Liron Yatziv, and Chenyang Xu \\ Siemens Corporate Research, Princeton NJ, USA
}

\begin{abstract}
We propose a novel method to detect the current state of the quasi-periodic system from image sequences which in turn will enable us to synchronize/gate the image sequences to obtain images of the organ system at similar configurations. The method uses the cumulated phase shift in the spectral domain of successive image frames as a measure of the net motion of objects in the scene. The proposed method is applicable to $2 \mathrm{D}$ and $3 \mathrm{D}$ time varying sequences and is not specific to the imaging modality. We demonstrate its effectiveness on X-Ray Angiographic and Cardiac and Liver Ultrasound sequences. Knowledge of the current (cardiac or respiratory) phase of the system, opens up the possibility for a purely image based cardiac and respiratory gating scheme for interventional and radiotherapy procedures.
\end{abstract}

\section{Introduction}

Image synchronization and gating are problems faced during the imaging and subsequent processing of organs with quasi-periodic motion, like the heart. The two most common sources of organ motion during imaging are respiratory and cardiac motion. The deformations caused by cardiac and respiratory motion make it difficult to image organs in the thorax and the abdomen. This severely limits the efficacy and efficiency of interventional and radiotherapy procedures performed in this region.

Different approaches have been devised to overcome cardiac and respiratory motion. Cardiac motion is usually handled by ECG gating whereas respiratory motion is usually handled by the use of markers placed on the patients body [1]. The problem with these approaches is that the ECG requires additional hardware, long setup times and in most cases there is a delay between the ECG signal and the image acquisition which makes it hard to make them synchronized. As far as the detection of respiratory phase is concerned, the placement of markers is usually impractical in a clinical setting; furthermore it is difficult to set up and prolongs the overall acquisition procedure. In addition most laboratories and existing image databases do not contain either ECG or respiratory information. Additionally, since the end goal is to perform image gating, detecting the phase using the images should be more reliable and robust compared to cases where an external signal is used. 
Another strategy for reducing respiratory motion is to acquire images using breath-hold techniques [2]. Although this reduces breathing motion by relatively simple and natural means, it is, nonetheless, restricted by the patient's ability to perform a supervised breath hold during the treatment [3]. A third class of strategies addresses the problem of respiratory motion correction by incorporating suitable motion models. Manke et al. 4] proposed a linear parametric model describing the relation between the variation of the diaphragmatic position and the respiratory-induced motion derived from image-intensity based registration for cardiac-triggered 3D MR imaging. King et al. [5] present an affine model which is based on the tracked motion of the diaphragm to compensate for respiratory motion in real-time X-ray images. The main drawback of these approaches is that they require manual landmark selection for diaphragm tracking.

Related Work. Prior work in this area is fairly limited, with [6] presenting an approach for retrospective gating of Intra coronary ultrasound (ICUS) sequences using feature extraction and classification. The method is computationally expensive and requires processing the whole sequence together as some of the features are temporal. In [7], the authors propose a method to analyze images in the sequence and retrieve the cardiac phase by using average image intensity and absolute image difference between the consecutive frames. The method is not very robust and has not been shown to work with real datasets. In [8], the authors propose to detect the respiration (phase) using mutual information. The mutual information (MI) is calculated between the fluoroscopic image and a reference angiogram. The MI calculation is performed only on selected regions of interest (ROI) and the results are variable depending on the choice of ROIs. A similar approach using Normalized MI is presented in [9]. A review of the effects of respiratory motion is presented in [10] and highlights the importance and need for reliable detection of the respiratory phase, specifically for the target organ. In 11, Berbeco et al. detect the breathing phase information by analyzing the fluoroscopic intensity fluctuations in the lung. However, the approach requires the selection of region of interest(ROI) in a section of the middle of the lung that does not contain the tumor.

Contributions. We propose a novel method to detect the current state of the quasi-periodic system which in turn will enable us to synchronize/gate image sequences to obtain images of the organ system at similar configurations. The proposed method is applicable to $2 \mathrm{D}$ and $3 \mathrm{D}$ time varying sequences and all imaging modalities. We demonstrate its effectiveness on 2D X-Ray Angiographic and 3D Liver and intra-cardiac Ultrasound sequences.

\section{Methods}

Let $I_{t}(x, y)$ represent the image at time $t$ of a scene changing with time. According to the phase correlation technique [12, if we represent the Fourier transform of $I_{t}(x, y)$ by $\mathcal{F}(\xi, \eta)$, and assume that objects in the scene exhibit only translations, then for $I_{t+1}(x, y)=I_{t}\left(x-x_{t}, y-y_{t}\right)$, we have

$$
\mathcal{F}_{t+1}(\xi, \eta)=e^{-j 2 \pi\left(\xi x_{t}+\eta y_{t}\right)} \mathcal{F}_{t}(\xi, \eta) .
$$


By inverse transforming the ratio of the cross-power spectrum of $I_{t+1}$ and $I_{t}$ to its magnitude,

$$
\frac{\mathcal{F}_{t+1} \mathcal{F}_{t}^{*}}{\left\|\mathcal{F}_{t+1} \mathcal{F}_{t}^{*}\right\|}=\exp \left(-j 2 \pi\left(\xi x_{t}+\eta y_{t}\right)\right),
$$

we obtain a peak at $\left(x_{t}, y_{t}\right)$. If we consider a simple 1D example as shown in Figure 1, we have the original image represented by the blue sinusoidal curve, and applying a translation to the image produces a phase shift in the spectrum.

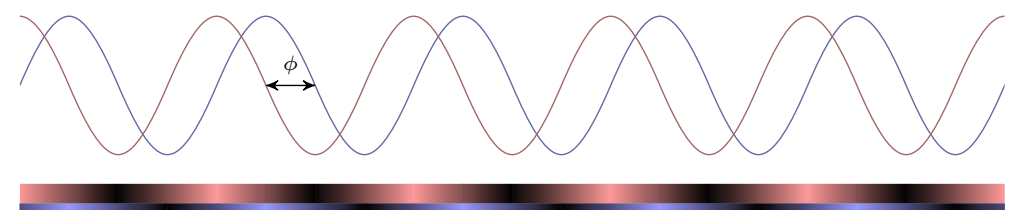

Fig. 1. The Relationship between object motion and the phase shift $(\phi)$

This approach was used in [13] to compensate for translational motion in coronary angiogram sequences.

It is important to realize that image features can be thought of as being a combination of signals at different frequencies, and that calculating the phase shift of the different frequencies in an image amounts to measuring the motion of image features corresponding to those frequencies. Measuring the phase shift in the frequency domain allows us to estimate the overall motion within an image without explicitly solving the problem of correspondence detection. The phase shift can be calculated in the Fourier domain [14. The overall change in the energy within the object being imaged can be estimated by integrating over the phase shift, which is the same as the energy change only due to the motion in the spatial domain because of Parsevals Theorem [14. It is important to note that this energy is not affected by changes in intensity which might happen as a result of varying levels of contrast agent and differing acquisition parameters. In addition, the proposed method is relatively insensitive to noise, as long as the spectrum of the noise is similar in successive images. As in this usually the case with image sequences acquired on the same scanner during the same acquisition session, the phase detection is robust to the noise levels during acquisition.

We estimate the energy change of the system being imaged by analyzing consecutive frames. The energy change in the system is computed in the spectral domain making the system more robust to outliers that might be introduced in the scene. Common examples are catheters, contrast agents, needles etc. The energy change in the scene is given by,

$$
E=\int \frac{\mathcal{F}\left(I_{t}\right)^{*} \cdot \mathcal{F}\left(I_{t+1}\right)}{\left|\mathcal{F}\left(I_{t}\right)^{*}\right|\left|\mathcal{F}\left(I_{t+1}\right)\right|} d f,
$$

where, $\mathcal{F}\left(I_{t}\right)$ represents the Fourier transform of the image $I$ at time $t$. 

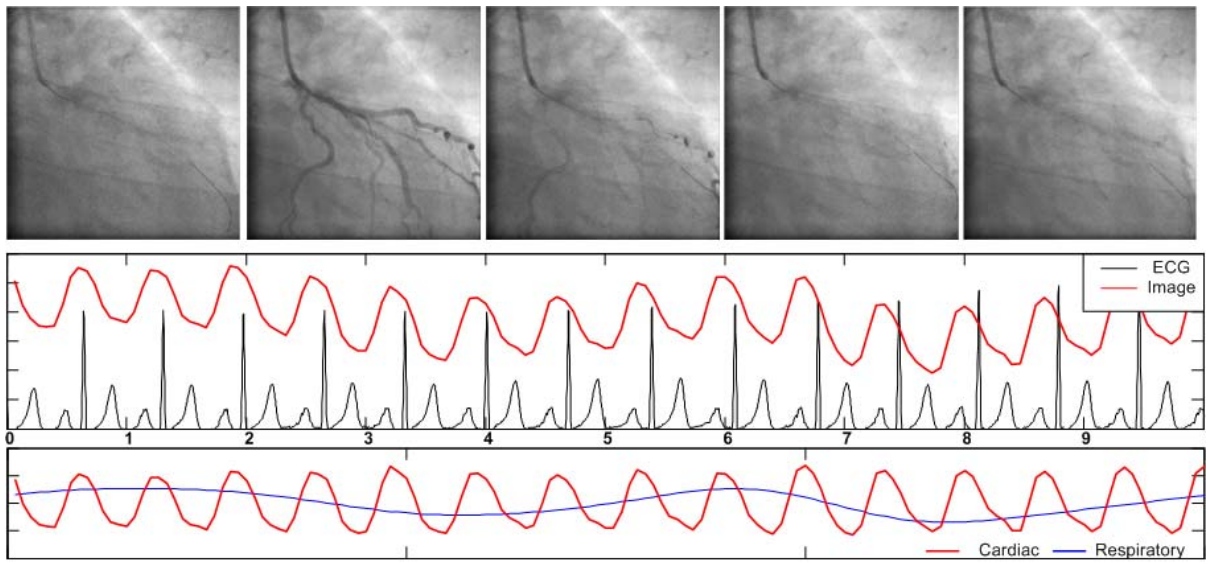

Fig. 2. Results of Phase detection in X-Ray sequences. The estimated phase is overlaid with the actual ECG signal. The low frequency variation in the phase corresponds to the respiratory cycle. The cardiac and respiratory cycles are also shown in this figure are separated using high and low pass filters respectively.

This energy is used as an estimate of the current phase of the organ. The extrema of this signal can be easily detected to detect the cardiac and the respiratory phases. Additionally, if separate cardiac and respiratory signals are required, then a band pass filter centered at $1 \mathrm{~Hz}$ should recover the cardiac signal, whereas a low pass filter will recover the respiratory signal. The system can be used with $N$-dimensional by computing the appropriate higher order Fourier transform.

\section{Results}

In order to test the efficacy and accuracy of the proposed method, we tested it on X-Ray and ultrasound(US) images and compared it against ground truth data. ECG signals were treated as ground truth for the detection of the cardiac cycle, and breathing phase obtained using a magnetic tracking system was treated as ground truth for respiratory motion.

Validation on Cardiac X-Ray Angiography images. We validate our method by comparing it against ECG signals embedded within 20 X-Ray angiographic and fluoroscopic images acquired on a biplane C-arm system (AXIOM Artis, Siemens Medical Solutions, Erlangen, Germany). The minima of the image-based phase correspond to end-diastole and therefore, we compared the alignment of this minima with the P-wave on the ECG signal. Additionally, for the cases where a mismatch existed, a visual comparison of images gated using the 2 methods yielded no obvious winner. An example of the detected phase overlaid on the ECG signal and with sample images is shown in Figure 2. Figure 2 also illustrates the separation of cardiac and respiratory signals from the combined phase signal. 


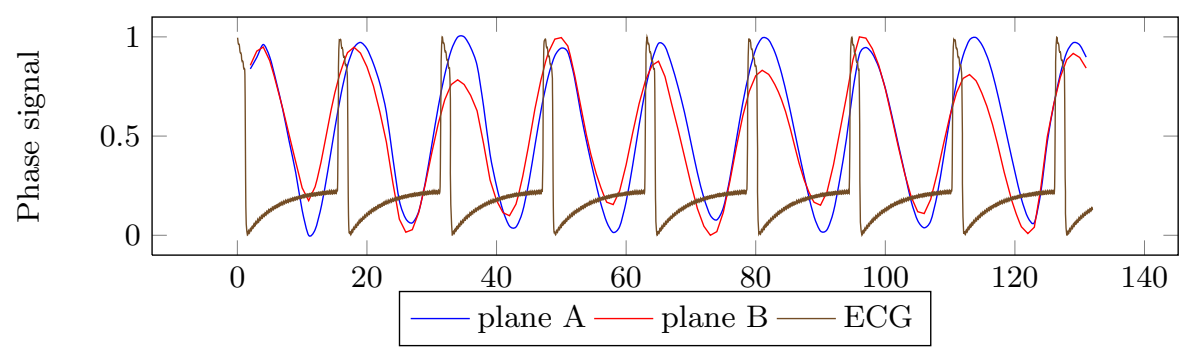

Fig. 3. Simple validation using biplane sequences. Normalize phase signals from a biplane Xray sequence are plotted along with the normalized ECG signal. It can clearly be seen that the phase is correctly calculated from both views independently.

We also compared the image-based phase signals from biplane X-Ray images. Since the two images are basically projections of the same heart, the phase signals should match, in spite of differences due to the projection angles. In Figure 3 we illustrate one such case in which the phase signals from both planes are plotted with the ECG QRS trigger signal. As can be seen, although there are small variations in the signals from the two planes, they are in overall agreement regarding the cardiac phase.

Validation on Liver 3D Freehand Ultrasound Images. In order to test the efficacy of the system on detecting respiratory motion, we used a Siemens ACUSON Sequoia 512 ultrasound machine (Siemens Ultrasound, Mountain View, CA) with abdominal curved-array probe, and a MicroBird magnetic tracking system (Ascencion Technology Corp., Burlington VT). "Tracked ultrasound" recordings were made in free breathing, with the transducer moving arbitrarily to image any longitudinal and transversal planes of the liver. A second MicroBird magnetic position sensor was attached to the chest and was tracked. The dominant translation of the tracked sensor was treated as a measure of the breathing phase [15. This was tested on four volunteers and in all cases the image based phase was in agreement with the tracked sensor. An example of this is shown in Figure 4]

Table 1. Correlation ratio of the detected phase against ground truth for angiography and liver ultrasound datasets

\begin{tabular}{|l|c|c|}
\hline Modality & Compared with & Correlation \\
\hline 2D Angiography & ECG & 0.92 \\
3D Liver US & Respiratory tracker & 0.88 \\
\hline
\end{tabular}

Validation on Intracardiac Echocardiography images. In order to test the image-based phase detection method on highly dynamic images, we applied it on Intracardiac Echocardiography (ICE) images acquired using a Siemens ACUSON $\mathrm{AcuNav}^{\mathrm{TM}}$ catheter 16. The images were acquired within the left atrium. Being inside the heart the image acquired by the ICE catheter changes rapidly and 


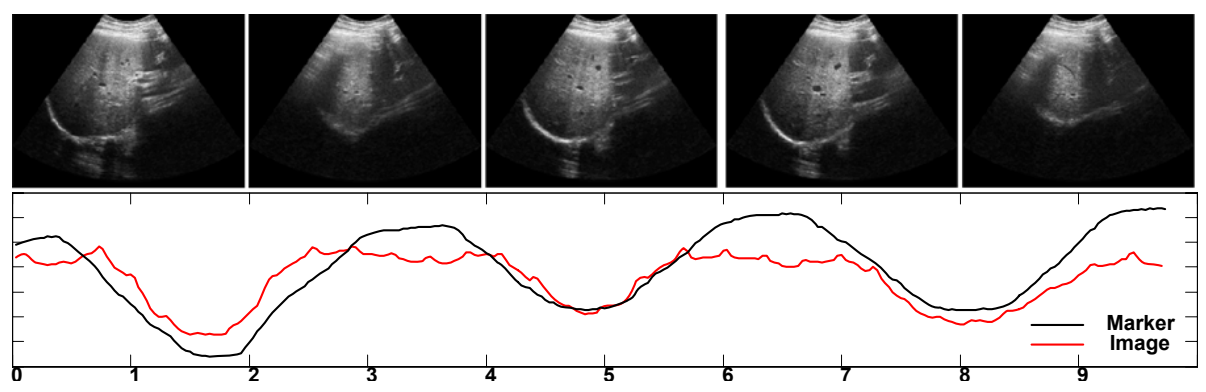

Fig. 4. Results of Phase detection in Liver ultrasound sequences. The estimated phase is overlaid with the dominant translation of a magnetic marker placed on the chest.

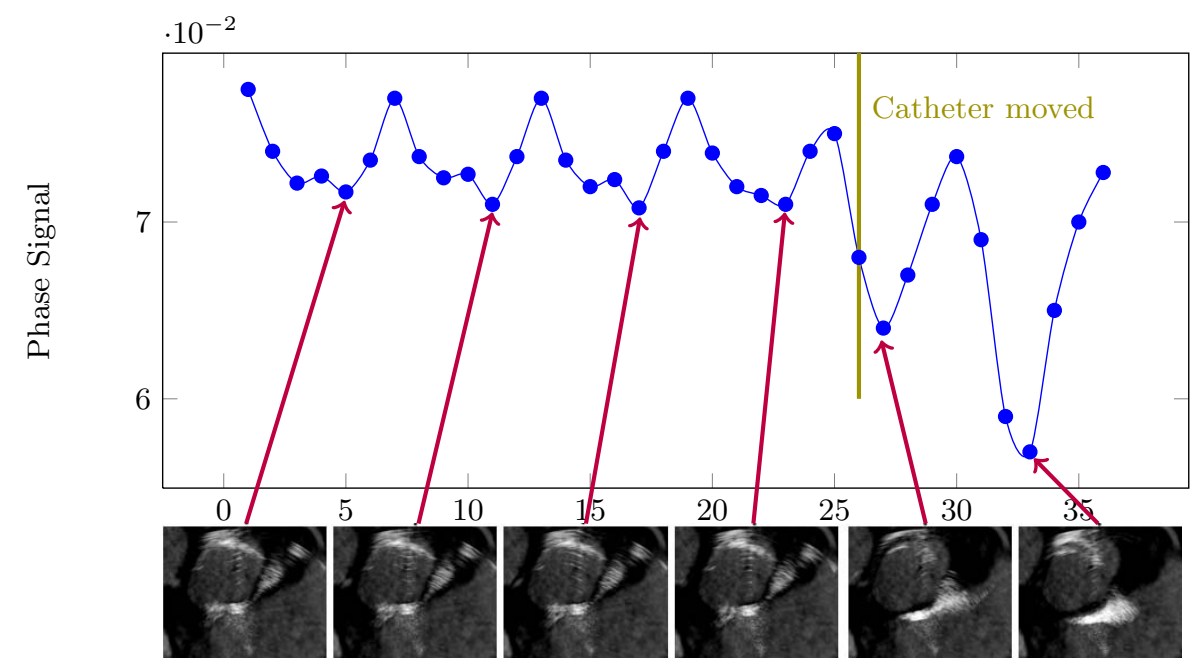

Fig. 5. Results of Phase detection in intra cardiac ultrasound sequences. The enddiastolic image are detected using the image-based phase detection algorithm. The figure also highlights the effect of a quick catheter move on the image-based phase signal.

is especially pronounced when the catheter is moved. The phase detection does however manage to detect the phase correctly, although in some cases there phase is lost when the catheter is moved rapidly. An example of the phase detection on ICE images is shown in Figure 5. The figure also illustrates the effect of a rapid catheter move.

\section{Implementation and Performance Considerations}

The major use of the proposed algorithm will be in real time interventional applications requiring a motion phase signal. Additionally, one of the main problems in using external triggers like ECG signals or displacement transducers is that 
the delay between the systems can be substantial and will limit the acquisition frequency. For any image based phase detection method to be practical, performance is as important as the accuracy with which the phase can be detected. The proposed algorithm is very fast, and we tested its performance using optimized code written using the Intel Integrated Performance Primitives for computing the Fourier transforms. We also tested using an implementation using nVidias CUDA library. The performance was evaluated on a Intel Core 2 Duo $2.2 \mathrm{GHz}$ notebook with a nVidia Quadro FX 5500 graphics card. We tested using a $1024 \times 1024$ angiographic sequence having 132 frames. The optimized software implementation took on an average 2.3 secs, implying a 60 fps capability with $1024 \times 1024$ images. The GPU implementation worked slightly faster on the same dataset, taking on an average 1.84 secs to process all 132 frames. Additionally, since the complexity of the proposed algorithm is dependent on Fourier transform of the input images, one easy way to speed up the algorithm further is to define regions of interest.

\section{Conclusion}

In this paper, we presented a novel algorithm for the automatic detection of the phase of a moving organ system directly from images. The algorithm is able to detect cardiac and respiratory phases from images of different imaging modality. We validated the algorithm on XRay angiographic, liver US and intra cardiac echocardiography images. ECG signals were used to validate the detection of the cardiac phase, and magnetic markers attached to the chest were used to validate the detection of the respiratory phase. The algorithm is very fast and can process images of size $1024 \times 1024$ at rates in excess of $60 \mathrm{fps}$.

The algorithm should be particularly useful in cardiac and abdominal interventional procedures where cardiac and respiratory motion make localization difficult and challenging. Current attempts at image fusion for such procedures are limited for these reasons. The availability of a reliable image-based phase detection algorithm should make it possible to compensate for intra-operative cardiac and/or respiratory motion. It is possible to use the signal for not only gating but also for real-time dynamic compensation. Real-time dynamic compensation is not currently available mainly because of the high computational complexity of most motion compensation algorithms. Since a pre-operative compensation for different phases of cardiac/respiratory motion can be performed, and the results simply recalled based on the current phase of the system, the image-based phase detection should be most useful for dynamic roadmapping applications for cardiac interventions.

\section{References}

1. Khamene, A., Warzelhan, J., Vogt, S., Elgort, D., Chefd Hotel, C., Duerk, J., Lewin, J., Wacker, F., Sauer, F.: Characterization of internal organ motion using skin marker positions. LNCS, pp. 526-533. Springer, Heidelberg (2004)

2. Paling, M.R., Brookeman, J.R.: Respiration artifacts in MR imaging: reduction by breath holding. J. Comput. Assist. Tomogr. 10(6), 1080-1082 (1986) 
3. Mageras, G.S., Yorke, E.: Deep inspiration breath hold and respiratory gating strategies for reducing organ motion in radiation treatment. Semin. Radiat. Oncol. 14(1), 65-75 (2004)

4. Manke, D., Rosch, P., Nehrke, K., Bornert, P., Dossel, O.: Model evaluation and calibration for prospective respiratory motion correction in coronary MR angiography based on 3-d image registration. IEEE Trans. on Medical Imaging 21(9), 1132-1141 (2002)

5. King, A.P., Boubertakh, R., Rhode, K.S., Ma, Y.L., Chinchapatnam, P., Gao, G., Tangcharoen, T., Ginks, M., Cooklin, M., Gill, J.S., Hawkes, D.J., Razavi, R.S., Schaeffter, T.: A subject-specific technique for respiratory motion correction in image-guided cardiac catheterisation procedures. Med. Image. Anal. 13(3), 419$431(2009)$

6. de Winter, S., Hamers, R., Degertekin, M., Tanabe, K., Lemos, P., Serruys, P., Roelandt, J., Bruining, N.: A novel retrospective gating method for intracoronary ultrasound images based on image properties. Computers in Cardiology, 13-16 (September 2003)

7. Zhu, H., Oakeson, K., Friedman, M.: Retrieval of cardiac phase from IVUS sequences. In: Proceedings of SPIE, vol. 5035, pp. 135-146 (2003)

8. Martin-Leung, B., Eck, K., Stuke, I., Bredno, J., Aach, T.: Mutual information based respiration detection. In: International Congress Series, vol. 1256, pp. 10851092. Elsevier, Amsterdam (2003)

9. Moser, T., Biederer, J., Nill, S., Remmert, G., Bendl, R.: Detection of respiratory motion in fluoroscopic images for adaptive radiotherapy. Physics in Medicine and Biology 53(12), 3129-3145 (2008)

10. Webb, S.: Motion effects in (intensity modulated) radiation therapy: a review. Physics in Medicine and Biology 51(13), 403 (2006)

11. Berbeco, R., Mostafavi, H., Sharp, G., Jiang, S.: Towards fluoroscopic respiratory gating for lung tumours without radiopaque markers. Physics in Medicine and Biology 50(19), 4481 (2005)

12. Kuglin, C., Hines, D.: The phase correlation image alignment method. In: Proc. Int. Conf. on Cybernetics and Society, vol. 4, pp. 163-165 (1975)

13. Wu, Q., Bones, P., Bates, R.: Translational motion compensation for coronary angiogram sequences. IEEE Transactions on Medical Imaging 8(3), 276-282 (1989)

14. Kaplan, W.: Advanced Calculus, 4th edn. Addison-Wesley, Reading (1992)

15. Wein, W., Cheng, J.Z., Khamene, A.: Ultrasound based respiratory motion compensation in the abdomen. In: MICCAI 2008 Workshop on Image Guidance and Computer Assistance for Soft-Tissue Interventions (September 2008)

16. Wein, W., Camus, E., John, M., Diallo, M., Duong, C., Al-Ahmad, A., Fahrig, R., Khamene, A., Xu, C.: Towards guidance of electrophysiological procedures with real-time 3d intracardiac echocardiography fusion to c-arm ct. In: Yang, G.-Z., et al. (eds.) MICCAI 2009, Part I. LNCS, vol. 5761, pp. 9-16. Springer, Heidelberg (2009) 